\title{
Bioarqueología de poblaciones prehistóricas de la quebrada de Humahuaca (Jujuy, Argentina)
}

Verónica SELdes ${ }^{1}$

\section{RESUMEN}

A partir de las caracterizaciones realizadas sobre los pueblos prehispánicos de la quebrada de Humahuaca, proponemos una serie de expectativas sobre el estilo de vida y el estado de salud de las poblaciones que habitaron la región en los períodos Formativo Tardio (500-900 DC), Desarrollos Regionales I (900-1250 DC) y II (1250-1430 $D C)$. Considerando que la bioarqueología constituye una vía importante para la realización de inferencias sobre los procesos de cambio social en las sociedades prehispánicas, se utilizan indicadores de estrés nutricional, estrés funcional, dieta y traumas para caracterizar cada período y evaluar las modificaciones en el tiempo en lo referido a patrones de subsistencia, tipos de recursos consumidos y estado de salud de las poblaciones.

Palabras claves: Quebrada de Humahuaca bioarqueología - estilo de vida - cambio social.

\section{ABSTRACT}

Through bioarchaeological characterizations of the prehispanic people of the Humahuaca valley, this paper suggests their lifestyle expectations and health status during the Late Formative (500-900 AD), Regional Development I (900-1250 AD) and II (1250-1430 AD) periods. Bioarchaeology constitutes an important way to make inferences about processes of social change in prehispanic populations. In this study, nutritional stress, functional stress, diet and trauma indicators are used to characterize each period and evaluate changes through time regarding subsistence patterns, the type of resources consumed and the health status of these populations.

Key words: Humahuaca valley - bioarchaeology - lifestyle - social change.

Recibido: septiembre 2005. Aceptado: enero 2006.

\section{Introducción}

Desde los inicios de la disciplina, los trabajos arqueológicos realizados en la quebrada de

\footnotetext{
${ }^{1}$ Instituto Interdisciplinario Tilcara, Universidad de Buenos Aires. Belgrano 445, (4624) Tilcara, Jujuy, ARGENTINA. Email: vseldes@arnet.com.ar
}

Humahuaca focalizaron sus objetivos en la caracterización de las poblaciones y en la proposición de secuencias para los procesos de desarrollo y cambio social de la región. Estos trabajos se basaron en la forma y decoración de las vasijas cerámicas, en la arquitectura y en las estructuras funerarias - por citar algunos ejemplos- para tratar diferentes momentos de ocupación, desde el Formativo Tardío (500-900 DC) hasta el Período Hispano-indígena (1536 DC en adelante) (Debenedetti 1912; Boman 1923; Casanova 1936; Bennett et al. 1948; Lafón 1958/59; González y Pérez Gollán 1966; Madrazo y Ottonello 1966; Pérez Gollán 1968, 1973; Palma 1993; Nielsen 1996a, 1996b, 1997 y 2001; Rivolta 2005).

Por su parte, la bioarqueología, a partir de los años 90, se ha visto enriquecida con la incorporación de conceptos provenientes de corrientes teóricas como, por ejemplo, la arqueología crítica, las teorías de la agencia y de la práctica, lo que se relaciona con un cambio en las preguntas, hipótesis y expectativas sobre la organización social, política y económica, y los procesos de diferenciación social de las poblaciones prehistóricas (Boyd 1996; Seldes 2004). En este sentido, la bioarqueología constituye una herramienta fundamental para reconstruir el comportamiento y una vía importante para la realización de inferencias sobre los procesos de cambio social en las sociedades, sobre todo, considerando la posibilidad de combinar una serie de indicadores (estrés nutricional, estrés funcional, dieta y traumas) capaces de señalar modificaciones en el tiempo en lo referido a patrones de subsistencia, tipos de recursos consumidos y estado de salud de las poblaciones.

Se ha postulado que si a lo largo del desarrollo de las formaciones sociales se producen situaciones de cambios en la organización social y los patrones de subsistencia, de alguna manera esto afectará al estilo de vida de las poblaciones (Cohen y Armelagos 1984; Goodman et al. 1984; Larsen 1997, entre otros). Tomando como punto de par- 
tida los postulados de la bioarqueología acerca de la posibilidad de que estos procesos se vean reflejados en el estilo de vida de las poblaciones pasadas y de acuerdo a las caracterizaciones realizadas sobre las poblaciones que habitaron la quebrada de Humahuaca (considerando, además, que las mismas están en constante revisión), en este trabajo se pretende reflexionar y proponer una serie de expectativas sobre la dieta y el estado de salud para los diferentes períodos en la región, realizando a la vez inferencias sobre los cambios que podrían haberse producido en lo biológico a partir de los cambios en las esferas sociopolíticas (p.e., cambios en los patrones de subsistencia, en los tipos de recursos consumidos, en el acceso a determinados tipos de recursos).

Las principales inquietudes sobre estas problemáticas surgen a la hora de pensar cómo los cambios en la organización sociopolítica afectarían la vida cotidiana de los individuos y de qué manera esto se reflejaría en el registro bioarqueológico. A partir de estas interrogantes este trabajo constituye más bien un ejercicio de reflexión, que un conjunto de argumentos acabados, es decir, no una serie de reglas a confirmar en el registro, sino un conjunto de preguntas que permitan combinar los diferentes indicadores bioarqueológicos, algo que ya iniciaron hace algunos años Mendonça y Bordach para esta región (Mendonça et al. 1991, 1992, 1997 y 2002; Bordach et al. 1998 y 1999).

Tomamos a tal efecto una serie de trabajos bioarqueológicos realizados sobre muestras correspondientes a diferentes momentos de ocupación de la quebrada de Humahuaca (Figura 1), desde el Formativo Tardío hasta el Período de Desarrollos Regionales II; se realiza de forma preliminar y con carácter exploratorio una comparación entre las expectativas propuestas para cada período y los resultados obtenidos, a la vez que una serie de comentarios sobre el potencial de la bioarqueología para realizar inferencias sobre aspectos de la organización social y los procesos de cambio social.

Para caracterizar el modo de vida de los pueblos prehispánicos de la quebrada de Humahuaca, se

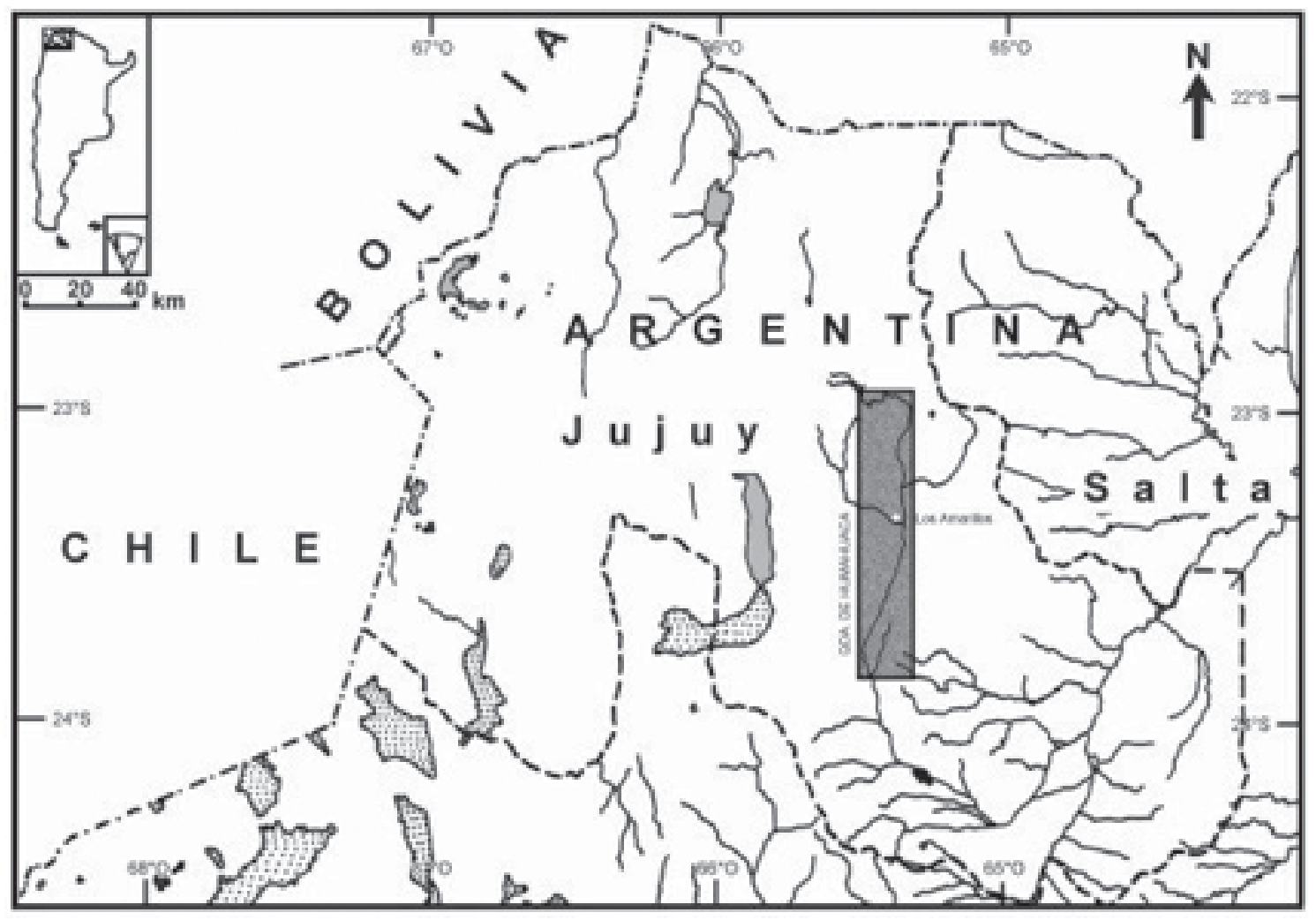

Figura 1. Ubicación de la quebrada de Humahuaca en Argentina. 
cuenta con poca información para los momentos anteriores a 1250 DC debido a las características del registro: los sitios, principalmente del Formativo Tardío, son difíciles de identificar por estar bajo aludes de barro y piedra o sepultados por las construcciones actuales. Las posibilidades de recuperar evidencia son escasas y la mayoría de las veces se trata de rescates realizados en viviendas particulares. En estos casos sólo es posible realizar excavaciones en marcos espaciales reducidos, lo que dificulta el análisis de las posibles asociaciones contextuales; por consiguiente, en muchas ocasiones es sumamente limitado el conocimiento disponible sobre los contextos funerarios. Diferente es el caso del Período de Desarrollos Regionales II (Nielsen 2001) o incluso del Incaico para el que se cuenta con mayor evidencia tanto arqueológica como bioarqueológica, y en el cual históricamente se centró la mayoría de los trabajos arqueológicos de la región.

\section{La quebrada de Humahuaca antes y después de $1250 \mathrm{DC}$}

Marcamos como punto de inflexión el 1250 DC para el proceso de desarrollo de las formaciones sociales de la quebrada de Humahuaca, porque se trata de un momento para el cual la mayoría de los investigadores coincide en que se produjeron cambios significativos en la forma de vida de los pueblos que la habitaron.

De acuerdo a las distintas caracterizaciones, podríamos resumir brevemente la forma de vida de estos pueblos en el Período Formativo Tardío y de Desarrollos Regionales I, de la siguiente manera (Nielsen 2001):

a) Emplazamiento en lugares bajos y accesibles en asociación directa con cursos de agua permanente y terrenos favorables para la agricultura.

b) Se trata de comunidades pequeñas, similares entre sí en las cuales no hay indicios de controles políticos centralizados o desigualdades sociales estructurales. No se esperan formas de especialización que excedan la división del trabajo al interior de la unidad doméstica.

c) Hacia el 900 DC (Período de Desarrollos Regionales I) se incrementa la cantidad de sitios y comenzaría una serie de cambios en lo referente al emplazamiento, trazado y distribución de los asentamientos: aumenta la densidad edilicia y las poblaciones se concentran en la quebrada troncal.

Hacia el 1250 DC las sociedades de la quebrada de Humahuaca se caracterizan por:

a) Nucleamiento de la población en conglomerados ubicados generalmente en lugares de gran visibilidad y difícil acceso.

b) Marcadas diferencias en el tamaño de los sitios y la presencia en alguno de ellos de espacios públicos, lo que llevó a plantear la existencia de jerarquías de asentamiento (Nielsen 1996b, 2001 y 2004).

c) Momento de gran concentración demográfica e integración política de carácter regional.

d) Cambios económicos, como intensificación agrícola y desarrollo de infraestructura hidráulica. En este período se activan lugares especializados en la producción agrícola, como El Alfarcito, Cosmate y Coctaca (Nielsen 1995).

e) Aumento en la explotación de los rebaños de llamas, inclusive en lo referido a fibra y transporte, y disminución de la importancia de la caza.

f) Surgimiento de formaciones políticas de una escala sin precedentes para el área, capaces de movilizar y coordinar gran cantidad de fuerza de trabajo.

Recientemente Nielsen (2004) propuso un modelo "corporativo" de organización social para las sociedades surandinas, tomando en consideración los trabajos de Blanton (1998); Blanton y colaboradores (1996), Feinmann (2000), McGuire y Saitta (1996) sobre las sociedades con características corporativas e incorporando nociones de la teoría de la práctica (Bourdieu 1977). De acuerdo a Nielsen (2004), estos sistemas se caracterizarían básicamente por:

a) Tener una marcada orientación corporativa, dotados de múltiples mecanismos institucionales que regularían el ejercicio del poder político y restringirían la acumulación económica por parte de individuos o linajes particulares.

b) Los ayllus mantendrían un control colectivo sobre los recursos económicos claves y retendrían 
el derecho de nombrar y fiscalizar sus propias autoridades.

c) Las principales formas de acumulación estarían basadas en capitales sociales y simbólicos; la verdadera riqueza del curaca no residiría en los bienes que poseía, sino en la magnitud de la red redistributiva que articulaba (Nielsen 2004).

\section{Expectativas bioarqueológicas}

De acuerdo a las interpretaciones que se han realizado sobre las formaciones sociales de la quebrada de Humahuaca se propone una serie de expectativas sobre los probables estilos de vida de los individuos en los diferentes momentos de ocupación de la región. No necesariamente se deben tomar como comportamientos o características a contrastar uno por uno en el registro, sino que los consideramos como puntos de partida para comenzar a reflexionar sobre las implicancias de las características de las sociedades sobre la biología humana.

Para las sociedades de los períodos Formativo Tardío y de Desarrollos Regionales I podemos postular las siguientes expectativas:

a) Podría esperarse que no haya diferencias entre los individuos en lo referido al acceso a los recursos y al estado de salud, ya que todos los individuos participarían de igual manera en las actividades productivas.

b) Si hay baja densidad demográfica, es esperable que los recursos sean suficientes para los niveles mínimos de subsistencia de los individuos.

c) Al tratarse de sociedades agropastoriles, la dieta estaría basada en recursos como el maíz, rico en carbohidratos y azúcares.

d) Podría pensarse que, por las características de las sociedades, la producción no excedería el nivel doméstico no generándose grandes cantidades de excedentes. Esto podría verse reflejado en una baja probabilidad de sobreexigencia física hacia los individuos.

De acuerdo a lo planteado para las sociedades del Período de Desarrollos Regionales II, podemos sugerir una serie de expectativas sobre el estilo de vida de los individuos, que en este caso constituyen principalmente preguntas iniciales, ya que se trata de propuestas teóricas recientes que requieren de una mayor reflexión:

- Si es a nivel del ayllu donde se manejan los recursos productivos podría esperarse que no haya un acceso diferencial de los recursos económicos y que los mismos estuvieran disponibles para toda la población.

- La producción excedería los requerimientos de la población siendo que el curaca acumula para su posterior redistribución hacia toda la población.

- Pero ¿qué sucedería si el curaca que en nombre del ayllu principal al cual pertenece acumula bienes productivos para la redistribución? Una posibilidad sería que él mismo consumiera alguna porción diferencial en la dieta.

- ¿La dieta de los individuos responderá a su inserción en las distintas esferas productivas? Los distintos grupos, inclusive a nivel interdoméstico, que se organicen en función de distintas tareas productivas ¿consumirán diferentes tipos de alimentos?

- Si los distintos grupos (p.e., pastores y agricultores) realizan diferentes tipos de tareas podría esperarse que las exigencias laborales sean diferenciales respondiendo al tipo de ocupación de cada uno.

- Las condiciones de vida (aglomeración de población en los pucaras) podrían estar generando una sobreexigencia para el desarrollo de algunas actividades cotidianas como, por ejemplo, transporte de agua y alimentos.

- La concentración poblacional también podría estar generando un deterioro en las condiciones de higiene, lo que podría facilitar la emergencia de enfermedades y epidemias, algo que ya ha sido planteado en la literatura arqueológica para Teotihuacán (Storey 1985).

- Las situaciones de violencia social que, de acuerdo a las interpretaciones del registro arqueológico se darían con grupos foráneos, podrían generar eventos de tipo traumático que repercutirían en la salud de los individuos a través de fracturas y golpes.

Estas expectativas pueden resumirse en el Cuadro 1 que permite visualizar las diferencias esperables en los diferentes períodos de ocupación: 


\begin{tabular}{|c|c|c|}
\hline & $\begin{array}{l}\text { Formativo Tardío } \\
\text { y Desarrollos Regionales I }\end{array}$ & Desarrollos Regionales II \\
\hline Acceso a los recursos & Sin diferenciación & $\begin{array}{l}\text { Sin diferenciación o sólo en el caso } \\
\text { del curaca }\end{array}$ \\
\hline Disponibilidad de recursos & Suficiente & Suficiente \\
\hline Producción excedentaria & No & Sí \\
\hline Sobreexigencia física & Baja & $\begin{array}{l}\text { Alta y diferencial por tipo de tarea } \\
\text { realizada }\end{array}$ \\
\hline Condiciones de higiene & Buenas & Malas \\
\hline Violencia social & Baja & Alta \\
\hline
\end{tabular}

Cuadro 1. Comparación de expectativas para los diferentes períodos de ocupación.

\section{Muestra}

Los restos óseos del Período Formativo Tardío provienen de rescates realizados en los sitios SJTil 20 y Flores-1, en el casco urbano de la localidad de Tilcara. De esto deriva que los trabajos fueron realizados en áreas acotadas y que en algunos casos no haya sido posible ampliar los límites de la excavación más allá de donde se hallaron los restos. La falta de perspectiva en cuanto a las estructuras funerarias y su asociación con otros tipos de estructuras dificulta la interpretación de las características arquitectónicas y organizacionales de los sitios. Por otra parte, para el Período de Desarrollos Regionales I se trabaja con restos recuperados del sitio Muyuna, mientras que para el Período de Desarrollos Regionales II las muestras provienen de los sitios Hornillos y Los Amarillos (Figuras 2 y 3).

Sitio SJTil 20: En el domicilio de la familia Mulqui, Mendonça y Bordach recuperaron restos inhumados en entierros individuales y múltiples, directos y en cámaras sepulcrales, mientras que algunos párvulos fueron inhumados en el interior de urnas (Mendonça et al. 1991 y 2002; Bordach et al. 1999). De acuerdo a los materiales recuperados en asociación con los restos humanos, el sitio fue asignado al Formativo Tardío, siendo los materiales analizados por los investigadores que realizaron la exhumación.

Sitio Flores-1: De una pequeña área excavada en el domicilio de la familia Flores se recuperaron restos humanos y materiales de un pequeño sector del lote debido a la presencia de otras construcciones actuales en el lugar. Estos materiales permiten adscribir los restos al Formativo Tardío. Los restos fueron exhumados por el equipo de arqueología de rescate del Instituto Interdisciplinario Tilcara.

Sitio Muyuna: Asentamiento residencial ubicado sobre una terraza fluvial alta en el margen izquierdo del río Grande. Nielsen excavó el sitio el año 2000 y recuperó un conjunto de individuos exhumados en seis unidades funerarias. El sitio es asignable al Período de Desarrollos Regionales I, con fechas radiocarbónicas que se distribuyen entre el 900 y el 1250 DC (Nielsen com. pers. 2000). Si bien la muestra se encuentra en mal estado de conservación, al ser la única conocida para este momento de ocupación, se rescata su valor como fuente de información y conocimiento para el período en cuestión.

Sitio Hornillos: El sitio arqueológico conocido como "Pucara de Hornillos" se ubica en el sector medio de la quebrada de Humahuaca. En el marco del Proyecto de la Secretaría de Cultura de la Provincia de Jujuy "Puesta en Valor del Patrimonio Cultural de Hornillos", Mamani (2001) realizó un rescate arqueológico de un contexto funerario, compuesto de dos áreas de entierro, limitadas por un muro simple de piedras; se trata de dos entierros individuales en urna, uno directo y uno múltiple y directo.

Sitio Los Amarillos: Conglomerado residencial prehispánico de estructura interna muy compleja que cubre 10 ha de superficie. Posee sectores de edificación bien diferenciados y una jerarquía de espacios públicos. Su mayor desarrollo parece haber tenido lugar durante la época inmedia- 


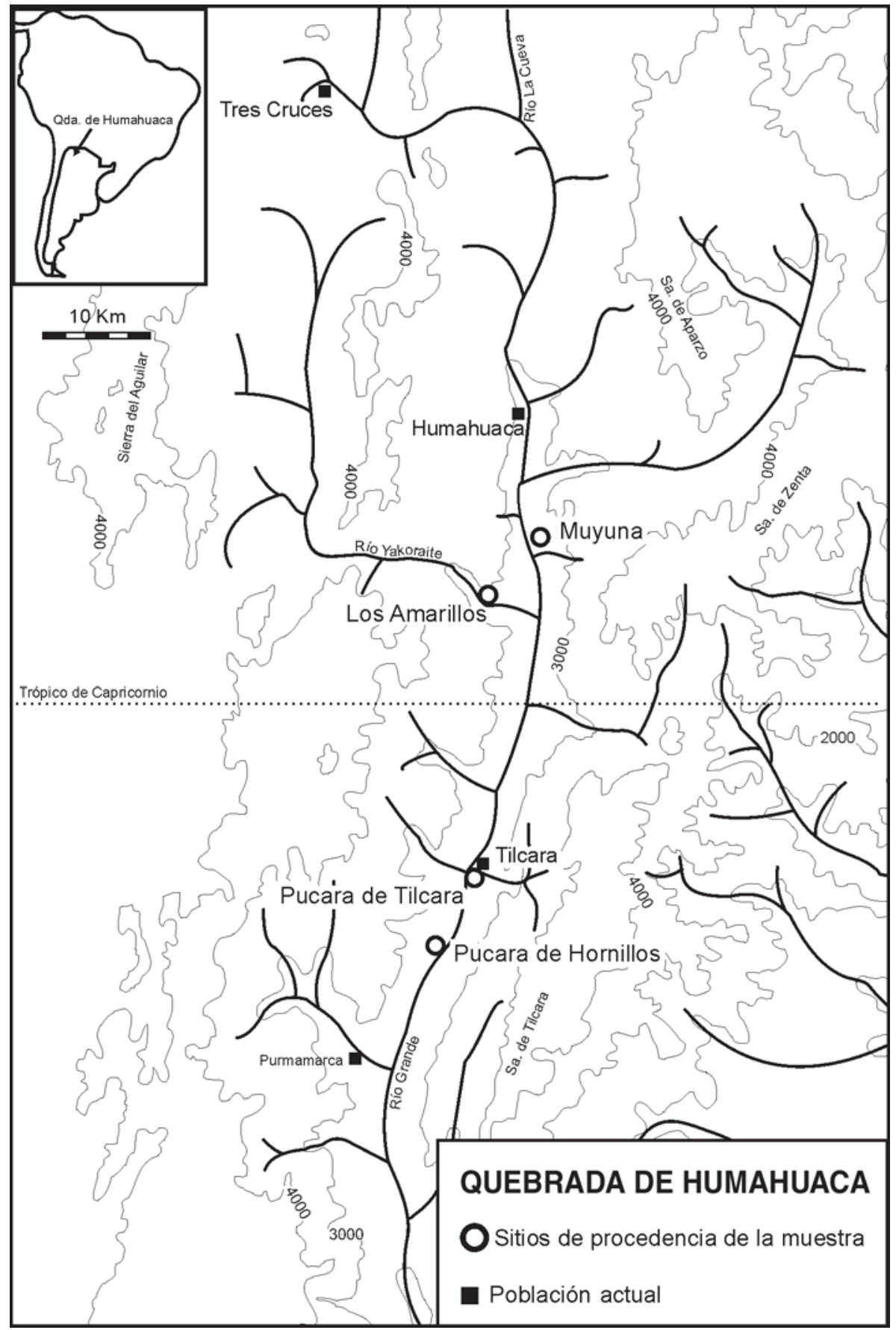

Figura 2. Procedencia de las muestras.

tamente anterior a la ocupación incaica. Las muestras provienen de dos complejos habitacionales de carácter doméstico recuperados en excavaciones sistemáticas. El Complejo E está localizado en un sector residencial periférico del asentamiento y fue trabajado por Nielsen y
Avalos; los restos provienen de tres recintos conectados entre sí, el recinto 301 en el cual se encontró un entierro múltiple y secundario en olla; el recinto 302 que consta de un entierro múltiple y secundario en una estructura que puede ser tanto una cista como otra estructura 


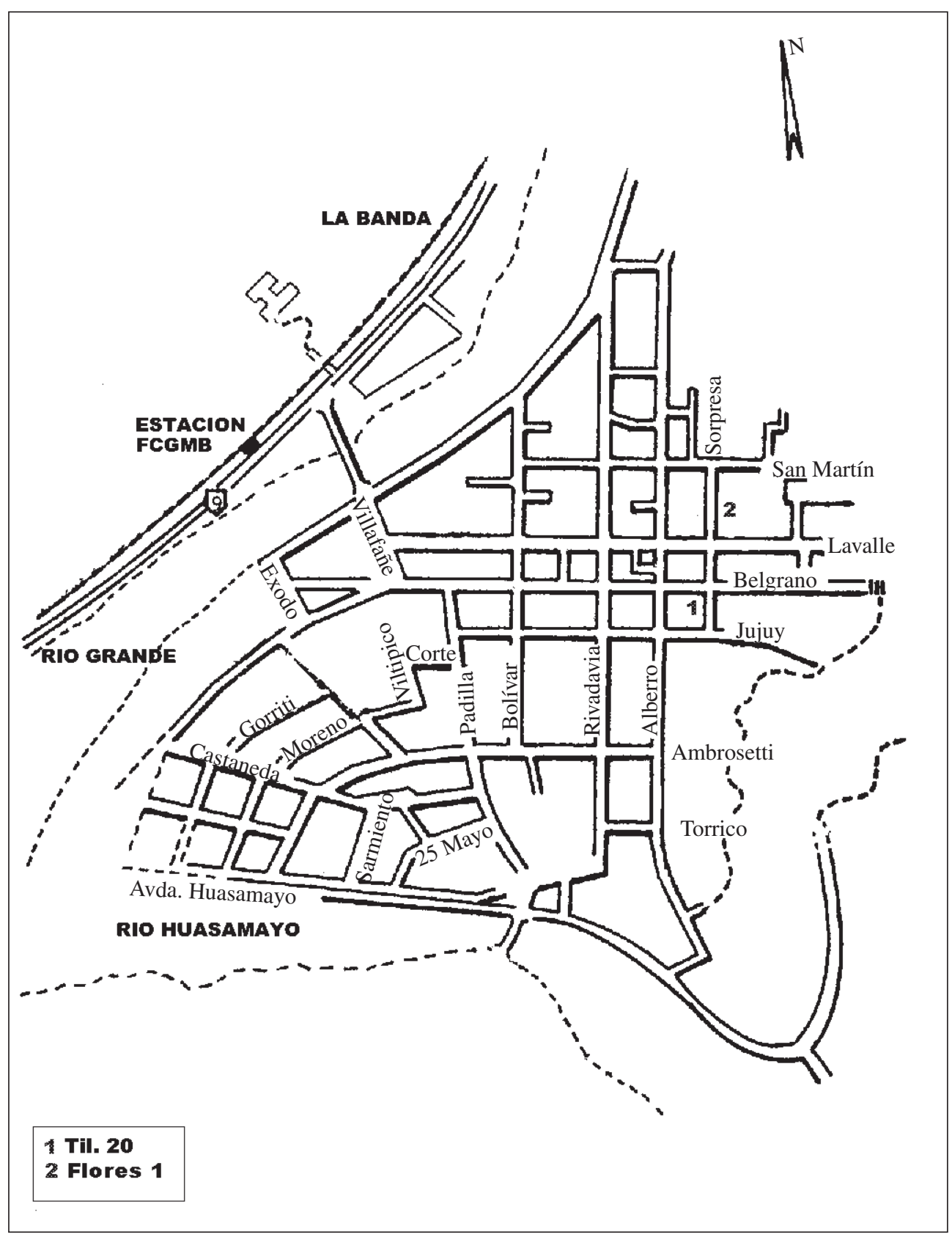

Figura 3. Ubicación de los sitios en la planta urbana de Tilcara.

reutilizada y, por último, el recinto 303 que contiene dos eventos de inhumaciones directos en pozo sin preparación previa; el primer evento (entierro 2) contiene dos individuos mientras que el segundo evento (entierro 1) contiene tres individuos. Por otra parte, la Unidad 400, trabajada por Taboada y Angiorama (2003a y 2003b), contenía tres cistas localizadas en el recinto 402; la cista 1 de carácter semisubterráneo contenía dos individuos; la cista 2 consistía en un entierro múltiple con varios eventos de inhumación; fue construida una vez abandonada la unidad como lugar de habitación, y por último, la cista 3 era subterránea y contenía dos individuos. 


\section{Metodología}

Los restos de SJTil 20 fueron analizados por Mendonça y colaboradores (1991, 1992, 2002) para determinar la edad utilizaron Todd (1921), Suchey y Katz (1986) y Brooks y Suchey (1990), y el sexo a partir de Buikstra y Ubelaker (1994).

Respecto de los demás conjuntos osteológicos, la determinación de edad sobre los restos humanos se realizó a partir de los estándares de Buikstra y Ubelaker (1994) y Brooks y Suchey (1990) para adultos, Scheuer y Black (2000) para infantiles y subadultos, y Fazekas y Kosa (1978) para perinatos. ${ }^{2} \mathrm{El}$ sexo, al igual que en el caso de SJTil 20, se determinó a partir de la morfología craneana y de la pelvis utilizando los estándares de Buikstra y Ubelaker (1994).

La estructura de la muestra de cada uno de los períodos se detalla en los cuadros 2 y 3 :
La muestra se analizó a partir de una serie de indicadores que permiten aproximarse al estilo de vida (Cohen y Armelagos 1984; Goodman et al. 1984; Iscan y Kennedy 1989) de los individuos aquí representados:

Estrés nutricional. ${ }^{3}$ En el proceso de interacción entre el organismo, el medio ambiente en el que viven y la organización social de la cual forman parte, los individuos pueden verse expuestos a situaciones que resulten críticas para los niveles mínimos necesarios para la subsistencia; se trata de situaciones que provocan desequilibrios que afectan la situación nutricional de los individuos y, por consiguiente, su estado de salud; es posible mencionar desde factores naturales como constricciones medioambientales (sequías, desvíos de los cursos de los ríos) a culturales (talvez el ejemplo más conocido en la literatura etnográfica sean las prohibiciones religiosas hacia el consumo de determinados alimentos) (Goodman et al. 1988).

\begin{tabular}{|llcccccc|}
\hline Individuos/Sitios & \multicolumn{2}{l}{ SJTil 20 (Mulqui) } & \multicolumn{2}{c}{ Flores-1 } & Muyuna & Total \\
\hline Perinato & 0 & 0 & 0 & 0 & 7 & $38.88 \%$ & $\mathbf{7}$ \\
Infantil & 3 & $21.43 \%$ & 2 & $33.33 \%$ & 2 & $11.11 \%$ & $\mathbf{7}$ \\
Subad. femenino & 0 & 0 & 1 & $16.66 \%$ & 0 & 0 & $\mathbf{1}$ \\
Subad. masculino & 0 & 0 & 1 & $16.66 \%$ & 0 & 0 & $\mathbf{1}$ \\
Adulto masculino & 5 & $35.71 \%$ & 0 & 0 & 3 & $16.66 \%$ & $\mathbf{8}$ \\
Adulto femenino & 6 & $42.86 \%$ & 1 & $16.66 \%$ & 5 & $27.77 \%$ & $\mathbf{1 2}$ \\
Adulto indet. & 0 & 0 & 1 & $16.66 \%$ & 1 & $5.55 \%$ & $\mathbf{2}$ \\
\hline Total & $\mathbf{1 4}$ & $\mathbf{1 0 0 \%}$ & $\mathbf{6}$ & $\mathbf{1 0 0 \%}$ & $\mathbf{1 8}$ & $\mathbf{1 0 0 \%}$ & $\mathbf{3 8}$ \\
\hline
\end{tabular}

Cuadro 2. Estructura de la muestra de los períodos Formativo Tardío y Desarrollos Regionales I.

\begin{tabular}{|c|c|c|c|c|c|c|c|}
\hline \multirow{2}{*}{$\begin{array}{l}\text { Individuos/Sitios } \\
\text { Perinato }\end{array}$} & \multicolumn{2}{|c|}{ Hornillos } & \multicolumn{2}{|c|}{$\begin{array}{c}\text { Los Amarillos } \\
\text { Complejo E }\end{array}$} & \multicolumn{2}{|c|}{$\begin{array}{c}\text { Los Amarillos } \\
400\end{array}$} & \multirow{2}{*}{$\begin{array}{r}\text { Total } \\
4\end{array}$} \\
\hline & 0 & 0 & 3 & $21.43 \%$ & 1 & $4.76 \%$ & \\
\hline Infantil & 4 & $50 \%$ & 5 & $35.71 \%$ & 7 & $33.33 \%$ & 16 \\
\hline Subadulto indet. & 0 & 0 & 1 & $7.14 \%$ & 0 & 0 & 1 \\
\hline Adulto Masculino & 0 & 0 & 2 & $14.29 \%$ & 4 & $19.05 \%$ & 6 \\
\hline Adulto Femeninos & 2 & $25 \%$ & 2 & $14.29 \%$ & 2 & $9.52 \%$ & 6 \\
\hline Adulto indet. & 2 & $25 \%$ & 1 & $7.14 \%$ & 6 & $28.57 \%$ & 9 \\
\hline Maduro Femenino & 0 & 0 & 0 & 0 & 1 & $4.76 \%$ & 1 \\
\hline Total & 8 & $100 \%$ & 14 & $100 \%$ & 21 & $100 \%$ & 43 \\
\hline
\end{tabular}

Cuadro 3. Estructura de la muestra. Período Desarrollos Regionales II.

2 Se definieron cinco intervalos de edad: Perinato: cercano al nacimiento hasta los seis meses de edad; Infantil: de seis meses a 10 años; Subadulto: de 10 a 20 años; Adulto: 20 a 30 años; Maduro: más de 30 años.
3 Se entiende por estrés a la disrupción fisiológica del organismo producto de una alteración del medio ambiente; el grado en que afecte a los individuos variará en función de la severidad del agente estresor y de la respuesta del propio organismo (Huss-Ashmore et al. 1982). 
Entre los indicadores de este tipo de estrés se utilizaron la hiperostosis porótica y criba orbitalia relacionadas con la presencia de anemia por la escasez en el consumo de proteínas cárnicas, aunque en realidad para explicar la presencia de esta patología se mencionan múltiples factores estresores actuando al mismo tiempo: anemia, infecciones crónicas, presencia de fitatos en el maíz que inhiben la absorción de hierro y, por último, presencia de parásitos en el organismo (Larsen 1997).

También se relevó la presencia de líneas de hipoplasia del esmalte que representan una disrupción en la amelogénesis o procesos de formación del esmalte dental e indican eventos de detención en el crecimiento. Debido a que el esmalte dental es muy sensitivo a los cambios metabólicos del organismo, su desarrollo puede alterarse por anomalías hereditarias, traumas, presencia de parásitos o eventos estresores (Buikstra y Ubelaker 1994); en este sentido se considera que no es posible identificar un estresor específico como factor causal de las líneas de hipoplasia, aunque se reconoce que las mismas están fuertemente relacionadas con el estado nutricional del individuo (Boyd 1996).

La presencia de indicadores de estrés nutricional en poblaciones prehispánicas se explica no por una causa única, sino que se desarrollan por una combinación de factores que incluyen constricciones medioambientales, biológicas (parásitos en el organismo, diarrea, infecciones) (Stini 1969), culturales (deficientes condiciones higiénicas) y sociales (desigual distribución de los recursos, agregación poblacional) (Martín et al. 1985), aunque se dice que el factor predominante lo constituyen las deficiencias nutricionales (Boyd 1996).

Dieta. La morbilidad de una población está directamente relacionada con la salud bucal de cada individuo y esta salud depende, en parte, del tipo de dieta que consumen los individuos a lo largo de su vida (Powell 1985); un bajo nivel de higiene bucal es causal de inflamaciones gingivales, abcesos, caries, y en última instancia, pérdida dental. En este trabajo se consideran a las caries y el desgaste del esmalte dental como indicadores de dieta.

Caries: Son consideradas patologías producto tanto de factores exógenos (minerales en suelo y agua, materiales abrasivos que contaminan la comida y composición química, textura, métodos de preparación y forma de consumo de los alimentos, higiene oral) como endógenos (agentes patógenos o bacterias, morfología de las coronas dentales, integridad del esmalte, composición química de la saliva y desgaste dental) (Powell 1985; Hillson 1996; Larsen 1997).

Desgaste del esmalte dental: El desgaste dental o atrición es considerado un proceso erosivo natural del esmalte, resultado del proceso masticatorio cuyo grado de desgaste aumenta con la edad. El grado y tipo de desgaste están fuertemente influidos por los tipos de alimentos que componen la dieta, los métodos de preparación de los mismos (partículas de los artefactos de molienda mezclados con los alimentos), y el uso que se haga de los dientes como herramientas (Molnar 1971; Hillson 1996).

Estrés funcional. Los indicadores de estrés funcional nos aproximan a las exigencias y constricciones impuestas tanto por el medio ambiente como por la propia organización social, y nos permiten inferir los patrones de actividades rutinarias desarrolladas por los individuos; esta área es importante, porque provee interesantes herramientas para la realización de inferencias sobre la organización social y económica de los pueblos prehistóricos (Larsen 1987).

Se consideró la osteoartritis ${ }^{4}$ o enfermedades degenerativas, ya que su formación en ciertas articulaciones del cuerpo depende de una serie de factores sistémicos -sexo, edad, metabolismo, nutrición, hereditarios, hormonales- y mecánicofuncionales; estos últimos se relacionan con las exigencias que producen las actividades que los individuos desarrollan en su vida cotidiana y que resienten las articulaciones y las degeneran (Jurmain 1977 y 1980). Se la considera reflejo de las actividades cotidianas realizadas por los individuos a la vez que producto de la organización del trabajo que caracteriza a esa población (Neves 1984).

Traumas. Estos indicadores ofrecen importante información sobre el comportamiento de las po-

\footnotetext{
${ }^{4}$ Se dividió la osteoartritis en cuatro grados: leve, moderada, intensa y "picos de loro".
} 
blaciones pasadas, ya que puede analizarse la recurrencia de ciertos patrones de patologías para determinar si se trata de accidentes fortuitos en el desarrollo de actividades cotidianas, o si, por el contrario, se deben a agresiones interpersonales, lo que nos acerca al grado de violencia social (Larsen 1987 y 1997).

Muchos de los traumas que sufre un individuo no son identificables a nivel osteológico y, por lo tanto, no se registran en el análisis bioarqueológico. Pero, sin embargo, el estudio de los casos observables presenta un gran potencial de información, porque permiten realizar inferencias sobre la organización social y las situaciones de conflicto de la población a la cual pertenece ese individuo (Larsen 1997). Entre los indicadores de traumas se pueden mencionar las fracturas, golpes e inflamaciones óseas producto tanto de violencia ejercida por un tercero como de accidentes.

\section{Resultados}

Tomamos muestras osteológicas de diferentes sitios arqueológicos correspondientes a los tres períodos para los que se conoce mayor cantidad de trabajos bioarqueológicos. Nuestro objetivo es principalmente considerar los resultados a la luz de las expectativas planteadas más que caracterizar a las poblaciones en sí mismas.

Respecto del registro bioarqueológico previo a 1250 DC, el Cuadro 4 indica que son bajas las frecuencias de patologías asociadas a eventos de estrés nutricional, ya que no se registraron casos para las líneas de hipoplasia del esmalte, mientras que un $36.36 \%$ presenta hiperostosis porótica o criba orbitalia; esto nos permitiría inferir que los individuos no estuvieron expuestos a eventos estresores generalizados -como lo indica el primer indicador señalado- ni específicos (esto último debido a la baja frecuencia de individuos con anemia).

Los indicadores de dieta señalan una alta frecuencia de caries (66.66\%), lo que permite hablar de una dieta basada en alimentos ricos en carbohidratos. En el caso del desgaste del esmalte dental, el alto porcentaje de casos de grado medio e intenso $(82.61 \%)$ podría señalar el consumo involuntario de materiales abrasivos junto a los alimentos como, por ejemplo, residuos de las piedras de moler mezclados con los alimentos molidos.

En cuanto al estrés ocupacional, las expectativas de encontrar bajas frecuencias de estos tipos de indicadores no se verían reflejadas totalmente en el registro bioarqueológico, ya que cerca de la mitad de los individuos ha sufrido de osteoartritis (47.37\%), lo que permite inferir un alto grado de esfuerzo corporal comprometido en el desarrollo de las actividades cotidianas.

También se esperaban bajas frecuencias en los traumas, y en este sentido es lo que se observa en

\begin{tabular}{|lcccccccc|}
\hline Indicador/Indiv. por sitio & \multicolumn{2}{c}{ SJTil 20 } & \multicolumn{2}{c}{ Flores-1 } & \multicolumn{2}{c|}{ Muyuna } & \multicolumn{2}{c|}{ Total } \\
\hline $\begin{array}{l}\text { ESTRES NUTRICIONAL } \\
\text { Hipoplasia esmalte }\end{array}$ & $0 / 8$ & 0 & $0 / 3$ & 0 & 0 & 0 & $0 / 11$ & 0 \\
Hiperostosis porótica & $3 / 8$ & $37.5 \%$ & $1 / 3$ & $33.33 \%$ & 0 & 0 & $4 / 11$ & $36.36 \%$ \\
$\begin{array}{l}\text { DIETA } \\
\text { Caries }\end{array}$ & $6 / 9$ & $66.66 \%$ & $0 / 4$ & 0 & $8 / 8$ & $100 \%$ & $14 / 21$ & $66.66 \%$ \\
Desgaste del esmalte & $10 / 10$ & $100 \%$ & $3 / / 4$ & $75 \%$ & $6 / 9$ & $66.66 \%$ & $19 / 23$ & $82.61 \%$ \\
FUNCIONAL & & & & & & & & \\
Osteoartritis & $6 / 8^{5}$ & $\mathbf{7 5 \%}$ & $0 / 4$ & 0 & $3 / 7^{6}$ & $42.86 \%$ & $9 / 19$ & $47.37 \%$ \\
TRAUMAS & $\mathbf{2 / 1 1}$ & $\mathbf{1 8 . 1 8} \%$ & $\mathbf{1 / 4}$ & $\mathbf{2 5 \%}$ & $\mathbf{2 / 8}$ & $\mathbf{2 5 \%}$ & $\mathbf{5 / 2 3}$ & $\mathbf{2 1 . 7 4 \%}$ \\
\hline
\end{tabular}

Cuadro 4. Indicadores bioarqueológicos. Períodos Formativo Tardío y Desarrollos Regionales I.

\footnotetext{
5 Principalmente en columna vertebral de grado medio.
}

6 Principalmente en columna vertebral y extremidades superiores de grado medio. 


\begin{tabular}{|c|c|c|c|c|c|c|c|c|}
\hline Indicador/Indiv. por sitio & \multicolumn{2}{|c|}{ Hornillos } & \multicolumn{2}{|c|}{$\begin{array}{l}\text { Los Amarillos } \\
\text { Complejo E }\end{array}$} & \multicolumn{2}{|c|}{$\begin{array}{l}\text { Los Amarillos } \\
\text { Unidad } 400\end{array}$} & \multicolumn{2}{|c|}{ Total } \\
\hline Hipoplasia esmalte & $0 / 4$ & 0 & $3 / 12$ & $25 \%$ & $4 / 13$ & $30.77 \%$ & $7 / 29$ & $24.14 \%$ \\
\hline Hiperostosis porótica & $2 / 4$ & $50 \%$ & $1 / 10$ & $10 \%$ & $8 / 16$ & $50 \%$ & $11 / 30$ & $36.66 \%$ \\
\hline $\begin{array}{l}\text { DIETA } \\
\text { Caries }\end{array}$ & $3 / 5$ & $60 \%$ & $5 / 13$ & $38.46 \%$ & $6 / 10$ & $60 \%$ & $14 / 28$ & $50 \%$ \\
\hline Desgaste del esmalte & $4 / 5$ & $80 \%$ & $4 / 8$ & $50 \%$ & $7 / 10$ & $70 \%$ & $15 / 23$ & $65.22 \%$ \\
\hline $\begin{array}{l}\text { FUNCIONAL } \\
\text { Osteoartritis }\end{array}$ & $3 / 4^{7}$ & $75 \%$ & $4 / 6^{8}$ & $66.66 \%$ & $14 / 14^{9}$ & $100 \%$ & $21 / 24$ & $87.50 \%$ \\
\hline TRAUMAS & $4 / 4$ & $100 \%$ & $2 / 4$ & $50 \%$ & $2 / 13$ & $15.38 \%$ & $8 / 21$ & $38.10 \%$ \\
\hline
\end{tabular}

Cuadro 5. Indicadores bioarqueológicos. Período de Desarrollos Regionales II.

los restos humanos: la muestra presenta un $21.74 \%$ de casos de fracturas y golpes, siendo factible inferir que la población prácticamente no sufrió de eventos traumáticos.

En cuanto al registro bioarqueológico después de 1250 DC, si bien de acuerdo a las expectativas planteadas esperábamos que no hubiera acceso y acumulación diferencial de los recursos económicos y que era probable que entre el curaca y el resto de la población sí lo hubiera, para poder comprobar esto último tendría que localizarse la unidad residencial y funeraria de aquellos que detentaran el poder en nombre del ayllu principal, algo que no es posible realizar en el marco de este trabajo.

En este caso, los indicadores de estrés nutricional (Cuadro 5) indican que son bajas las frecuencias de casos de hipoplasia del esmalte $(24.14 \%)$ y de hiperostosis porótica $(36.66 \%)$, con lo cual se podría inferir que los individuos no estuvieron sometidos a eventos estresores, o que no tuvieron dificultades en acceder a los recursos necesarios para su subsistencia, de tal manera que resultaran perjudiciales para su estado de salud.

La pregunta de si los individuos o grupos dedicados a distintas tareas productivas consumirían diferentes tipos de alimentos, no es posible de responder con la muestra aquí considerada, ya que

7 Principalmente en columna vertebral y extremidades superiores y de grado medio a intenso.

8 De grado medio e intenso en columna vertebral, extremidades superiores e inferiores. hasta el momento no hemos podido distinguir unidades residenciales con particulares tareas productivas. Lo que sí se observa en la muestra es que la dieta indica que la población consumía altos componentes de carbohidratos, ya que la mitad muestra casos de caries (50\%); nuevamente son altos los porcentajes de individuos con grados medios a intensos de desgaste del esmalte dental (65.22\%). En la Unidad 400 se registraron casos de individuos de edad infantil con un desgaste pronunciado del esmalte dental asociado probablemente al uso de la dentadura como herramienta.

Respecto a los indicadores de estrés funcional, habíamos planteado que, de distinguir unidades residenciales dedicadas a trabajos específicos (p.e., pastoreo, agricultura), se esperaría que presentaran diferentes grados de exposición corporal de acuerdo a las demandas de cada tipo de actividad laboral. Esto tampoco ha sido posible de analizar con la muestra disponible; a su vez, por las propias condiciones de vida, los individuos sufrirían una sobreexigencia que resultaría perjudicial para su estado de salud. En este caso se registraron altos porcentajes de individuos de edad adulta con osteoartritis $(87.50 \%)$ sin encontrar diferencias entre individuos de diferente sexo, lo que indica que también en este período los individuos realizaron actividades que significaron grandes esfuerzos para el organismo.

Las situaciones de conflicto social que podrían generarse producirían traumas en el organismo. 9 De grado medio e intenso en columna vertebral, extremi-
dades superiores e inferiores. 
En el caso de este tipo de indicadores, las frecuencias de traumas, fracturas y golpes son bajas $(38.10 \%)$, no pudiendo inferirse situaciones de violencia a partir del registro bioarqueológico.

\section{Expectativas, registro bioarqueológico y organización social}

En general, los resultados estarían enmarcados en lo esperado para cada período, aunque es necesario reflexionar sobre el grado en que los datos provistos por la bioarqueología se ajustan a las expectativas y, a la vez, de qué manera se complementan con las propuestas de desarrollo y cambio social generadas a partir de registros arqueológicos diferentes.

Si bien la bioarqueología puede dar cuenta de alguna manera de los cambios sociales (Larsen 1997), en nuestro caso sólo es posible trabajar a nivel muy preliminar, principalmente por el grado de conocimiento de las sociedades formativas de la quebrada de Humahuaca. En cambio, es posible ver si hay un correlato de los cambios en el registro bioarqueológico, algo ya trabajado por Costin y Earle (1989) al evaluar los cambios que se produjeron en el patrón de consumo de la población Wanka producto del proceso de dominación del Imperio Incaico, y por Hastorf (1990) para los cambios producidos por la sociedad Sausa, también producto de la conquista incaica.

Los cambios a nivel estructural pueden significar en las prácticas cotidianas modificaciones en la frecuencia y tipos de recursos consumidos, en la intensidad de trabajo invertido o en las condiciones de salubridad e higiene, esto debería verse reflejado en el tipo de dieta consumida por los individuos o en el grado de sobrexigencia corporal. Para evaluar a nivel osteológico dichos cambios, se realiza una comparación entre las muestras de los diferentes momentos de ocupación esperando observar diferencias en cuanto a la salud y a la nutrición de los individuos.

De acuerdo a los resultados (Cuadro 6), no se observan diferencias estadísticamente significativas en el estilo de vida y el estado de salud a lo largo del desarrollo de las formaciones sociales de la quebrada de Humahuaca, es decir, que los cambios que se registran a otros niveles (p.e., estructuras de los sistemas de asentamientos, em-

\begin{tabular}{|lrc|}
\hline Indicador/ Período & $\begin{array}{r}\text { Antes de } \\
\mathbf{1 2 5 0} \text { DC }\end{array}$ & $\begin{array}{c}\text { Después de } \\
\mathbf{1 2 5 0} \text { DC }\end{array}$ \\
\hline $\begin{array}{l}\text { ESTRES NUTRIC. } \\
\text { Hipoplasia esmalte }\end{array}$ & $0 \%$ & $24.14 \%$ \\
Hiperostosis & $36.36 \%$ & $36.66 \%$ \\
$\begin{array}{l}\text { DIETA } \\
\text { Caries }\end{array}$ & $66.66 \%$ & $50 \%$ \\
$\begin{array}{l}\text { Desgaste } \\
\text { FUNCIONAL }\end{array}$ & $82.61 \%$ & $65.22 \%$ \\
Osteoartritis & $47.37 \%$ & $87.50 \%$ \\
TRAUMAS & $21.74 \%$ & $38.10 \%$ \\
\hline
\end{tabular}

Cuadro 6. Comparación entre los períodos.

plazamiento de los sitios, forma y decoración de las vasijas cerámicas) no se verían reflejados en las muestras analizadas.

Si bien la cantidad de individuos que hemos considerado es baja, por lo que este trabajo debe ser visto como una primera aproximación, resultan interesantes las tendencias observadas hacia una importante presencia de estrés ocupacional en los diferentes períodos y una mayor presencia en el Período de Desarrollos Regionales II, lo que estaría indicando que la sobreexigencia física fue mayor que en períodos anteriores. Asimismo, es posible inferir que en los diferentes períodos de ocupación los individuos no han sufrido de eventos de estrés nutricional, que presentan altas frecuencias de caries vinculados a una dieta basada en recursos ricos en carbohidratos y azúcares, que el alto grado de desgaste del esmalte dental estaría relacionado con el consumo involuntario de materiales abrasivos y que, por último, no habrían estado expuestos a eventos traumáticos.

Sugerimos que para poder realizar interpretaciones vinculadas a los procesos sociales, como los propuestos para la quebrada de Humahuaca, deberían ampliarse las muestras de cada uno de los períodos. También el potencial del registro bioarqueológico se vería incrementado con la incorporación de otros indicadores como líneas de Harris para indicadores de estrés nutricional o isótopos estables para dieta (Larsen 1997); por último, es indispensable la integración con otras líneas de evidencia como la arqueofauna, la paleobotánica y el comportamiento mortuorio (Mercolli y Seldes 2005 Ms). 
Otra interpretación que debería considerarse es que los cambios registrados en ciertas esferas de la forma de vida de los pueblos prehispánicos no necesariamente implican cambios profundos en el estilo de vida y en las condiciones de salud de los individuos como para dejar marcas en los huesos. En este sentido, consideramos que cada registro debe tratarse de manera independiente, aunque sin aislarlo de su contexto, para evitar la realización de inferencias automáticas de una dimensión a otra.

Agradecimientos A Axel Nielsen y María Vázquez por sus sugerencias y comentarios, los resultados finales son de mi absoluta responsabilidad.

\section{REFERENCIAS CITADAS}

BENNETT, W., E. BLEILER y F. SOMMER, 1948. Northwest Argentine archaeology. Yale University Publications in Anthropology 38, New Haven.

BLANTON, R., 1998. Beyond centralization: Steps toward a theory of egalitarian behavior in Archaic States. En Archaic States, G. Feinmann y J. Marcus (Eds.), pp. 135172. School of American Research Press, Santa Fe.

BLANTON, R., G. FEINMANN, S. KOWALESKI y P. PEREGRINE, 1996. A dual-processual theory for the evolution of Mesoamerican Civilization. Current Anthropology 37: 1-14.

BOMAN, E., 1923. Los ensayos para establecer una cronología prehispánica en la región Diaguita (República Argentina). Boletín de la Academia Nacional de Historia VI: $1-31$.

BORDACH, M. A., O. MENDONÇA, M. RUIZ y M. E. ALBECK, 1998. El joven señor de La Falda: Indicadores de una persona social en el Tilcara Hispano-Indígena. En Los desarrollos locales y su territorio, B. Cremonte (Ed.), pp. 199-208. Universidad Nacional de Jujuy, San Salvador de Jujuy.

BORDACH, M. A., L. DALERBA y O. MENDONÇA, 1999. Vida y muerte en quebrada de Humahuaca. Antropología física prehistórica del sitio SJTil 20. Universidad Nacional de Río Cuarto, Río Cuarto.

BOYD, D., 1996. Skeletal correlates of human behavior in the Americas. Journal of Archaeological Method and Theory 3: 189-251.

BOURDIEU, P., 1977. Outline of a theory of practice. Cambridge University Press, Cambridge.

BROOKS, S. y J. SUCHEY, 1990. Skeletal age determination based on the os pubis: A comparison of the AcsadiNemeskeri and Suchey-Brooks methods. Human Evolution 5: 227-238.

BUIKSTRA, J. y D. UBELAKER, 1994. Standards for data collection from human skeletal remains. Arkansas Archeological Survey Resarch Series 44, Arkansas.
CASANOVA, E., 1936. La quebrada de Humahuaca. Historia de la Nación Argentina 1: 207-249. Junta de Historia y Numismática Americana, Buenos Aires.

COHEN, M. y G. ARMELAGOS (Eds.), 1984. Paleopathology at the origins of agriculture. Academic Press, Orlando.

COSTIN, C. y T. EARLE, 1989. Status distinction and legitimation of power as reflected in changing patterns consumption in Late Prehispanic Perú. American Antiquity 54 (4): 691-714.

DEBENEDETTI, S., 1912. Los cementerios prehistóricos de La Isla de Tilcara (Provincia de Jujuy). Actas del XVII Congreso Internacional de Americanistas, pp. 502-508. Buenos Aires.

FAZEKAS, M. y M. KOSA, 1978. Forensic fetal osteology. Akademiai Kiadó, Budapest.

FEINMANN, G., 2000. Corporate/network: New perspectives on modes of political action and the Puebloan Southwest. En Social theory in archaeology, M. Schiffer (Ed.), pp. 31-51. University of Utah Press, Salt Lake City.

GOODMAN, M., D. MARTIN, G. ARMELAGOS y G. CLARK, 1984. Indicators of stress from bone and teeth. En Paleopathology at the origins of agriculture, M. Cohen y G. Armelagos (Eds.), pp. 13-49. Academic Press, Nueva York.

GOODMAN, A., R. BROOKE THOMAS, A. SWED LUND y G. ARMELAGOS, 1988. Biocultural perspectives on stress in prehistoric, historical, and contemporary population research. Yearbook of Physical Anthropology 31: 169-202.

GONZALEZ, A. R. y J. A. PEREZ, 1966. El Area Andina Meridional. Actas del XXXVII Congreso Internacional de Americanistas, pp. 241-265. Sevilla.

HASTORF, C., 1990. The effect of the Inka State on Sausa agricultural production and crop consumption. American Antiquity 55 (2): 691-714.

HILlSON, S., 1996. Dental anthropology. Cambridge University Press, Cambridge. 
HUSS-ASHMORE, R., A. GOODMAN y G. ARMELAGOS, 1982. Nutritional inference from paleopathology. En Advances in archaeological method and theory, vol. 5, M. Schiffer (Ed.), pp. 395-473. Academic Press, San Diego.

ISCAN, M. y K. KENNEDY, 1989. Reconstruction of life from the skeleton. Alan R. Liss, Inc. Nueva York.

JURMAIN, R., 1977. Stress and etiology of osteoarthritis. American Journal of Physical Anthropology 46: 353- 366.

- 1980. The pattern of involvement of appendicular degenerative joint disease. American Journal of Physical Anthropology 53: 143-150.

LAFON, C., 1958/59. Ensayo sobre cronología e integración de la Cultura Humahuaca. Runa IX, partes 1-2.

LARSEN, C., 1987. Bioarcheological interpretation of subsistence economy and behavior from human skeletals remains. En Advances in archaeological method and theory, vol. 10, M. Schiffer (Ed.), pp. 339-445. Academic Press, San Diego.

- 1997. Bioarcheology. Interpreting behavior from the human skeleton. Cambridge University Press, Cambridge.

MADRAZO, G. y M. OTTONELLO, 1966. Tipos de instalación prehispánica en la región de la puna y su borde. Monografías 1, Museo Etnográfico Municipal "Dámaso Arce", Olavarría.

MAMANI, H. E., 2001. Puesta en valor del patrimonio cultural de Hornillos (Depto. de Tilcara, Provincia de Jujuy). Actas del XIV Congreso Nacional de Arqueología Argentina, pp 284-285. Rosario.

MARTIN, D., A. GOODMAN y G. ARMELAGOS, 1985. Skeletal pathologies as indicators of quality and quantity of diet. En The analysis of prehistoric diet, vol. 8, R. Gilbert y J. Mielke (Eds.), pp. 227-279. Academic Press, Orlando.

MCGUIRE, R. H. y D. J. SAITTA, 1996. Although they have petty captains, they obey them badly: The dialectics of prehispanic Western Pueblo social organization. American Antiquity 61: 197-216.

MENDONÇA, O., M. A. BORDACH, M. GROSSO y L. DALERBA, 1991. Nuevas evidencias del Período Agroalfarero Temprano en quebrada de Humahuaca. Los hallazgos del sitio Til 20 (Tilcara, Jujuy). Comechingonia 8 (7): $31-45$.

MENDONÇA, O., M. A. BORDACH y S. VALDANO, 1992. Reconstrucción del comportamiento biosocial en el Pucara de Tilcara (Jujuy). Una propuesta heurística. Cuadernos 3: $144-151$

MENDONÇA, O., M. A. BORDACH, M. E. ALBECK y M. RUIZ, 1997. Collares de vidrio y ollas de barro. Comportamiento ante la muerte en el Tilcara hispano-indígena inicial (Jujuy, Argentina). Cuadernos 9: 175-202.
2002. Ambiente, comunidad y comportamiento biosocial en el Formativo de Tilcara, quebrada de Humahuaca, Jujuy. Pacarina 2: 135-147.

MERCOLLI, P. y V. SELDES, 2005 Ms. ¿Señoríos o sociedades corporativas? Perspectivas desde los registros bioarqueológico y zooarqueológico. Ponencia presentada en el Taller Procesos Sociales Prehispánicos en los Andes Meridionales, Tilcara.

MOLNAR, S., 1971. Human tooth wear, tooth function and cultural variability. American Journal of Physical Anthropology 34: 175-189.

NEVES, W., 1984. Estilo de vida e osteobiografia: A reconstitusão de comportamento pelos ossos humanos. Revista Prehistoria 6: 287-291.

NIELSEN, A., 1995. Aportes al estudio de la producción agrícola Inca en la quebrada de Humahuaca (Jujuy, Argentina). Hombre y Desierto 9, T I, pp. 245-256.

-1996a. Demografía y cambio social en quebrada de Humahuaca (Jujuy, Argentina), 700-1535 DC. Relaciones de la Sociedad Argentina de Antropología XXI: 307385.

_ 1996b. Estructuras y jerarquías de asentamientos en Humahuaca (Jujuy, Argentina) en vísperas de la invasión europea. En XXV Aniversario Museo Arqueológico Dr. Eduardo Casanova, pp. 99-109. Instituto Interdisciplinario de Tilcara, Universidad de Buenos Aires, Tilcara.

-1997. Tiempo y cultura material en la quebrada de Humahuaca 700-1650 DC. Serie Monografías. Instituto Interdiscilpinario Tilcara, Facultad de Filosofía y Letras, Universidad de Buenos Aires, Tilcara.

2001. Evolución social en quebrada de Humahuaca (AD 700-1536). En Historia argentina prehispánica, vol. 1, E. Berberián y A. Nielsen (Eds.), pp. 171-264. Editorial Brujas, Córdoba.

2004. Pobres jefes: Aspectos corporativos en las formaciones sociales preincaicas de los Andes circumpuneños. En Contra el pensamiento tipológico: Reflexiones teóricas actuales sobre complejidad social, C. Langebaek y C. Gnecco (Eds.), Universidad de los Andes, Bogotá. En prensa.

PALMA, J., 1993. Aproximación al estudio de una sociedad compleja: Un análisis orientado en la funebria. Arqueología 3: 41-68.

PEREZ GOLLAN, J. A., 1968. Subárea de Humahuaca. Actas y Memorias del XXXVII Congreso Internacional de Americanistas, T 2, pp. 273-293. Buenos Aires

-1973. Arqueología de culturas agroalfareras de la quebrada de Humahuaca (Provincia de Jujuy, República Argentina). América Indígena XXXIII: 667-678.

POWELL, M., 1985. Dental wear and caries in dietary reconstruction. En The analysis of prehistoric diet, vol. 10, 
R. Gilbert y J. Mielke (Eds.), pp 307-337. Academic Press, Orlando.

RIVOLTA, M. C., 2005. Cambio social en la quebrada de Humahuaca (1100-1400 DC). Instituto Interdisciplinario Tilcara, Facultad de Filosofía y Letras, Universidad de Buenos Aires, Tilcara.

SCHEUER, L. y S. BLACK, 2000. Developmental juvenile osteology. Academic Press, San Diego.

SELDES, V., 2004. Bioarqueología social. Nuevas preguntas de investigación, nuevas perspectivas. Estudios Sociales del NOA 7: 67-91.

_2005 Ms. Análisis bioarqueológico sobre un conjunto de restos óseos humanos recuperados en el domicilio de la familia Flores, Tilcara.

STINI, W., 1969. Nutritional stress and growth: Sex difference in adaptive response. American Journal of Physical Anthropology 31: 417-426.
STOREY, R., 1985. An estimate of mortality in a precolumbian urban population. American Anthropologist 87: 519-535.

SUCHEY, J. y D. KATZ, 1986. Skeletal age standars derived from an extensive multirracial sample of modern Americans. Abstract. American Journal of Physical Anthropology 69: 269.

TABOADA, C. y C. ANGIORAMA, 2003a. Buscando los indicadores arqueológicos de la unidad doméstica. Cuadernos 20: 393-407.

2003b. Posibilidades de un enfoque dinámico para el estudio de la arquitectura doméstica prehispánica. Un caso de aplicación en Los Amarillos (Jujuy). Relaciones de la Sociedad Argentina de Antropología 28: 101-115.

TODD, T., 1921. Age changes in the pubic bone. I: The male white pubis. American Journal of Physical Anthropology 3: $285-334$ 